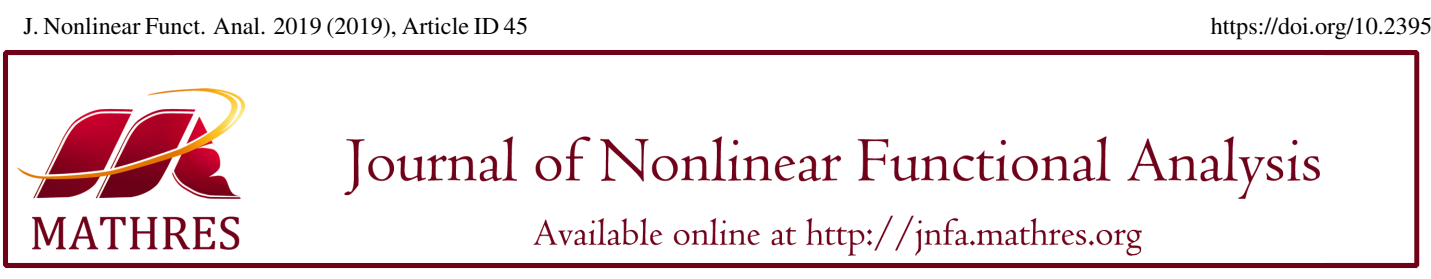

\title{
NEW EXISTENCE THEOREMS FOR VECTOR EQUILIBRIUM PROBLEMS WITH SET-VALUED MAPPINGS
}

\author{
TAO CHEN ${ }^{1}$, SHUFANG ZOU ${ }^{1}$, YU ZHANG $^{2, *}$ \\ ${ }^{1}$ School of Fundamental Sciences, Yunnan Open University, Kunming, 650223, China \\ ${ }^{2}$ College of Statistics and Mathematics, Yunnan University of Finance and Economics, Kunming 650221, China
}

\begin{abstract}
In this paper, based on the well-known Ky Fan section theorem and separation theorems, and new assumptions, some existence results on vector equilibrium problems and weak vector equilibrium problems with set-valued mappings are obtained, respectively. Some examples are given to illustrate our main results.
\end{abstract}

Keywords. Section theorem; Set-valued mappings; Separation theorem; Vector equilibrium problem.

2010 Mathematics Subject Classification. 65K05, 90C33.

\section{INTRODUCTION}

Let $X$ and $V$ be real Hausdorff topological vector spaces, and let $X_{0} \subset X$ be a nonempty subset. Let $S$ be a pointed closed convex cone. (Weak) Vector equilibrium problem is to find $\bar{x} \in X_{0}$ such that

$$
f(\bar{x}, y) \notin-S \backslash\{\theta\}(-i n t S), \quad \forall y \in X_{0},
$$

where $\theta$ is the zero element of $V$ and $f: X_{0} \times X_{0} \rightarrow V$ is a vector-valued mapping. This problem provides a unified framework of many important problems, such as, vector optimization problems, vector saddle point problems, vector non-zero sum game problems and vector variational inequality problems and has wide applications in mathematical economics and operational research; see, e.g., $[1,2,3,4,5,6]$ and the references therein. In view of these important applications, a lot of authors have devoted themselves to the study of existence results of vector equilibrium problems and weak vector equilibrium problems. Bigi, Capătă and Kassay [2] investigated existence theorems for vector equilibrium problems by virtue of separation theorems of convex sets. As a special case, they also obtained existence results for vector saddle point and vector variational inequalities. Ansari and Flores-Bazán [3] investigated the necessary and sufficient conditions of weakly vector equilibrium problems by using a recession method. By a

${ }^{*}$ Corresponding author.

E-mail addresses: chentao1cq@126.com (T. Chen), zsfct2000@126.com (S. Zou), zhangyu198606@sina.com (Y. Zhang).

Received May 19, 2019; Accepted November 5, 2019.

(C)2019 Journal of Nonlinear Functional Analysis 
vector various minimax theorem, Lin [4] obtained an existence result for generalized weak vector equilibrium problems. Under convex and nonconvex domains, Gong [5] investigated existence problems for weak vector equilibrium problems. Kien, Wong and Yao [6] investigated existence results for variational inequality problems under some pseudomonotone and discontinuity assumptions.

The payoff function of classic decision problems is single; a real number or a vector. However, it is difficult to know the exact values of payoffs owing to some unexpected situations. We can only give an estimate of values of payoffs. Therefore, decision problems with set payoff become an interesting research topic. Research on set-valued mappings is a new branch of mathematics. As a powerful tool for solving nonlinear analysis problems, it has become an indispensable part of nonlinear analysis; see, e.g., [7, 8, 9, 10, 11, 12, 13]. Li, Chen and Lee [7] and Li, Chen and Yang [8] first obtained set various minimax theorems. Zhang and Li [9] investigated some set various Ky Fan minimax theorems. Han and Huang [10] obtained the existence and connectedness results of solutions for weak vector quasiequilibrium problems with set-valued mappings.

Motivated by these results, we establish some existence theorems of solutions for two type of vector equilibrium problems with set-valued mappings under some new assumptions. Some examples are also given to illustrate our main results. The rest of the paper is organized as follows. In Section 2, we present notations and preliminary results. In Section 3, we state some existence theorems for set vector equilibrium problems and set weak vector equilibrium problems.

\section{PRELIMINARIES}

Let $X$ and $V$ be real Hausdorff topological vector spaces. Assume that $S$ is a pointed closed convex cone in $V$ with its interior int $S \neq \emptyset$. The dual cone $S^{*}$ of $S$ is defined as follows:

$$
S^{*}:=\left\{s^{*} \in V^{*}: s^{*}(s) \geq 0, \forall s \in S\right\},
$$

where $s^{*}(s)$ is the value of the continuous linear function $s^{*}$ on $s$.

First, we give the following two type of set various vector equilibrium problems. Let $F: X_{0} \times X_{0} \rightarrow 2^{V}$ be a set-valued mapping.

Set vector equilibrium problem (SVEP) is to find $\bar{x} \in X_{0}$ such that

$$
F(\bar{x}, y) \bigcap(-S \backslash\{\theta\})=\emptyset, \quad \forall y \in X_{0} .
$$

Set weak vector equilibrium problem (SWVEP) is to find: $\bar{x} \in X_{0}$ such that

$$
F(\bar{x}, y) \bigcap(-\operatorname{int} S)=\emptyset, \quad \forall y \in X_{0} .
$$

Some fundamental terminologies are presented as follows.

Definition 2.1. [14] Let $F: X \rightarrow 2^{V}$ be a set-valued mapping, and $x_{0} \in X$.

$F$ is said to be quasi-upper (lower) semicontinuous on $x_{0}$ iff, for any $b \in V$,

$$
F\left(x_{0}\right) \not \subset b+S\left(F\left(x_{0}\right) \not \subset b-S\right),
$$

there exists a neighborhood $U$ of $x_{0}$ such that

$$
F(x) \not \subset b+S(F(x) \not \subset b-S), \quad \forall x \in U .
$$

$F$ is said to be $S$-quasi-upper (lower) semicontinuous on $X$ iff $F$ is $S$-quasi-upper (lower) semicontinuous for any $x \in X$. 
Lemma 2.2. The set-valued mapping $F: X \rightarrow 2^{V}$ is $S$-quasi-upper semicontinuous on $X$ if and only if, for any $b \in V$, the level set

$$
\operatorname{Lev}_{F}(b):=\{x \in X: F(x) \subset b+S\}
$$

is closed.

Proof. From the proof of Lemma 2.2 in [14], we can obtain the desired immediately.

Definition 2.3. $[8,9,10]$ Let $X_{0}$ be a nonempty convex set in $X$ and let $F: X_{0} \rightarrow 2^{V}$ be a set-valued mapping.

(i) The set map $F$ is said to be properly $S$-quasiconvex iff, for any $x_{1}, x_{2} \in X_{0}$ and $l \in[0,1]$,

$$
F\left(x_{1}\right) \subset F\left(l x_{1}+(1-l) x_{2}\right)+S \quad \text { or } \quad F\left(x_{2}\right) \subset F\left(l x_{1}+(1-l) x_{2}\right)+S ;
$$

(ii) The set map $F$ is said to be $S$-quasiconvex iff, for any $z \in V$, the set

$$
\operatorname{Lev}_{F}(z):=\left\{x \in X_{0}: \exists t \in F(x) \text {, s.t. } t \in z-S\right\}
$$

is convex;

(iii) The set map $F$ is said to be natural $S$-quasiconvex iff, for any $x_{1}, x_{2} \in X_{0}$ and $l \in[0,1]$,

$$
\operatorname{co}\left\{F\left(x_{1}, F\left(x_{2}\right)\right)\right\} \subset F\left(l x_{1}+(1-l) x_{2}\right)+S .
$$

Next, we give the nonlinear scalarization functions and some useful lemmas.

Definition 2.4. [15] Let $k \in$ int $S$ and $a \in V$. The nonlinear scalarization functions $\xi_{k a}(z): V \rightarrow R$ is defined by

$$
\xi_{k a}(z)=\min \{t \in R: z \in a+t k-S\} .
$$

Lemma 2.5. [16] Let $k \in$ int $S$ and $a \in V$. The following conclusions hold:

(i) $\xi_{k a}(z) \leq r \Leftrightarrow z \in a+r k-S$;

(ii) $\xi_{k a}(z) \geq r \Leftrightarrow z \notin a+r k-$ int $S$;

(iii) $\xi_{k a}(\cdot)$ is strictly monotonically increasing $\left(\left(z_{1}-z_{2} \in \operatorname{int} S \Rightarrow \xi_{k a}\left(z_{1}\right)>\xi_{k a}\left(z_{2}\right)\right)\right)$;

(iv) $\xi_{k a}(\cdot)$ is monotonically increasing $\left(\left(z_{1}-z_{2} \in S \Rightarrow \xi_{k a}\left(z_{1}\right) \geq \xi_{k a}\left(z_{2}\right)\right)\right)$;

(v) $\xi_{k a}(\cdot)$ is continuous.

Lemma 2.6. Let $k \in$ int $S$ and $a \in V$. If the set map $F: X_{0} \rightarrow 2^{V}$ is properly $S$-quasiconvex, then $\xi_{k a} \cdot F$ : $X_{0} \rightarrow 2^{R}$ is $R_{+}$- quasiconvex.

Proof. We only need to prove that, for any $r \in R$, the set

$$
\operatorname{Lev}_{F}(r)=\left\{x \in X_{0}: \exists t \in \xi_{k a} \circ F(x) \text { s.t. } t \leq r\right\}
$$

is convex. Let $x_{1}, x_{2} \in \operatorname{Lev}_{F}(r)$ and $l \in[0,1]$. Thus, there exist $z_{1} \in F\left(x_{1}\right), z_{2} \in F\left(x_{2}\right)$ such that

$$
\xi_{k a}\left(z_{1}\right) \leq r, \quad \xi_{k a}\left(z_{2}\right) \leq r .
$$

By Lemma 2.5 (i), we have

$$
z_{1} \in a+r k-S, \quad z_{2} \in a+r k-S .
$$

Since $F$ is properly $S$-quasiconvex, there exists $z^{\prime}, z^{\prime \prime} \in F\left(l x_{1}+(1-l) x_{2}\right)$ satisfying $z^{\prime} \in z_{1}-S$ or $z^{\prime \prime} \in$ $z_{2}-S$. Hence,

$$
z^{\prime} \in a+r k-S
$$


or

$$
z^{\prime \prime} \in a+r k-S
$$

By Lemma 2.5 (i), we have $\xi_{k a}\left(z^{\prime}\right) \leq r$ or $\xi_{k a}\left(z^{\prime \prime}\right) \leq r$, that is,

$$
l x_{1}+(1-l) x_{2} \in \operatorname{Lev}_{F}(r) \text {. }
$$

Lemma 2.7. [9] Let $X_{0} \subset X$ be a nonempty convex set, and let $F: X_{0} \rightarrow 2^{R}$ be a set-valued map. The following two statements are equivalent:

(i) $\forall r \in R, \quad\left\{x \in X_{0}: \exists t \in F(x)\right.$ s.t. $\left.t \leq r\right\}$ is convex;

(ii) $\forall w \in R, \quad\left\{x \in X_{0}: \exists t \in F(x)\right.$ s.t. $\left.t<w\right\}$ is convex.

Lemma 2.8. [17, 18] Let $X_{0} \subset X$ be a nonempty convex subset, and let $A \subset X_{0} \times X_{0}$. If

(i) $\forall y \in X_{0},\left\{x \in X_{0}:(x, y) \in A\right\}$ is closed;

(ii) $\forall x \in X_{0},\left\{y \in X_{0}:(x, y) \notin A\right\}$ is convex or empty;

(iii) $\forall x \in X_{0},(x, x) \in A$,

then there exists $x_{0} \in X_{0}$ such that $\left\{x_{0}\right\} \times X_{0} \subset A$.

\section{MAIN RESULTS}

First, by using the Ky Fan section theorem and the non-convex separation theorem, we prove the following result.

Theorem 3.1. Let $k \in$ int $S$, and $a \in V$. Let $X_{0} \subset X$ be a nonempty compact convex subset, and let $F: X_{0} \times Y_{0} \rightarrow 2^{V}$ be a set map. If the following conditions are satisfied:

(i) for each $y \in X_{0}, F(x, y)$ is quasi $S$-upper semicontinuous and $F(x, x)$ is quasi S-lower semicontinuous;

(ii) for each $x \in X_{0}, F(x, \cdot)$ is properly $S$-quasiconvex on $X_{0}$;

(iii) $\bigcup_{x \in X_{0}} F(x, x) \subset V \backslash\{-S \backslash\{\theta\}\}$,

then (SVEP) has a solution.

Proof. We divide the proof into the following several steps.

Step 1 . We prove that there exists $\bar{x} \in X_{0}$ satisfying

$$
\xi_{k a} \circ F\left(\bar{x}, X_{0}\right) \subset \bigcup_{x \in X_{0}} \xi_{k a} \circ F(x, x)+R_{+} .
$$

Let

$$
A:=\left\{(x, y) \in X_{0} \times X_{0}: \xi_{k a} \circ F(x, y) \subset \bigcup_{x \in X_{0}} \xi_{k a} \circ F(x, x)+R_{+}\right\} .
$$

First, we show that, for any $y \in X_{0}$, the set $\left\{x \in X_{0}:(x, y) \in A\right\}$ is closed. Let $\left\{x_{n}\right\} \subset\left\{x \in X_{0}:(x, y) \in A\right\}$ and $x_{n} \rightarrow x_{0}$. By the assumptions, for any $y \in X_{0}$, we have

$$
\xi_{k a} \circ F\left(x_{n}, y\right) \subset \min \bigcup_{x \in X_{0}} \xi_{k a} \circ F(x, x)+R_{+} .
$$

By Lemma 2.2, for any $y \in X_{0}$, we have

$$
\xi_{k a} \circ F\left(x_{0}, y\right) \subset \min \bigcup_{x \in X_{0}} \xi_{k a} \circ F(x, x)+R_{+},
$$


that is,

$$
\left\{x \in X_{0}:(x, y) \in A\right\}
$$

is a closed set.

Second, we show that, for any $x \in X_{0},\left\{y \in X_{0}:(x, y) \notin A\right\}$ is convex. By using the definition, we have

$$
\left\{y \in X_{0}:(x, y) \notin A\right\}=\left\{y \in X_{0}: \exists z \in \xi_{k a} \circ F(x, y) \text { s.t. } z<\min \bigcup_{x \in X_{0}} \xi_{k a} \circ F(x, x)\right\} .
$$

From Lemmas 2.6 and 2.7, for each $x \in X_{0}$, we obtain that

$$
\left\{y \in X_{0}:(x, y) \notin A\right\}
$$

is convex. From the definition of $A$, it is obvious that $x \in X_{0},(x, x) \in A$. It follows from Lemma 2.8 that there exists $\bar{x} \in X_{0}$ such that

$$
\{\bar{x}\} \times X_{0} \subset A,
$$

i.e., (3.1) holds.

Step 2. We prove that there exists $\bar{x} \in X_{0}$ satisfying

$$
F\left(\bar{x}, X_{0}\right) \subset \bigcup_{x \in X_{0}} F(x, x)+S .
$$

As a matter of fact, if

$$
z \notin \bigcup_{x \in X_{0}} F(x, x)+S
$$

i.e.,

$$
(z-S) \bigcap\left(\bigcup_{x \in X_{0}} F(x, x)\right)=\emptyset,
$$

we find from Lemma 2.5 (i) that

$$
\xi_{k z}(u)>0, \quad \forall u \in \bigcup_{x \in X_{0}} F(x, x) .
$$

By (3.1), there exists $\bar{x} \in X_{0}$ such that

$$
\xi_{k z} \circ F\left(\bar{x}, X_{0}\right) \subset \operatorname{int} R_{+},
$$

that is,

$$
\xi_{k z}(w)>0, \quad \forall w \in F\left(\bar{x}, X_{0}\right) .
$$

Thanks to Lemma 2.5 (i), we obtain $z \notin w+S$. Hence,

$$
z \notin F\left(\bar{x}, X_{0}\right) .
$$

So, (3.2) holds.

Step 3. We show that (SVVEP) has a solution.

Since

$$
S+V \backslash\{-S \backslash\{\theta\}\}=V \backslash\{-S \backslash\{\theta\}\},
$$

We obtain from (3.2) and condition (iii) that there exists $\bar{x} \in X_{0}$ such that

$$
F\left(\bar{x}, X_{0}\right) \subset V \backslash\{-S \backslash\{\theta\}\},
$$

i.e.,

$$
F\left(\bar{x}, X_{0}\right) \bigcap(-S \backslash\{\theta\})=\emptyset .
$$


This shows that there exists $\bar{x} \in X_{0}$ such that

$$
F(\bar{x}, y) \bigcap(-S \backslash\{\theta\})=\emptyset, \quad \forall y \in X_{0} .
$$

Remark 3.2. (i) In the literatures, there are some existence results for the SVEP. However, the continuity assumption on map $f$ is related to the neither open nor closed set $S \backslash\{\theta\}$. Thus, it is difficult to verify the corresponding ones. Moreover, since the set $S \backslash\{\theta\}$ is neither closed nor open, the direct investigation on the existence results for the SVEP via traditional methods is not easy. Clearly, Theorem 3.1 is different from theirs.

(ii) In Step 2 of the proof above, we cannot obtain (3.2) by using the (strictly) monotonically increasing property of the nonlinear function $\xi_{k a}$. Meanwhile, it can be seen that the nonlinear function $\xi_{k a}$ depicts the property of the separation between a convex cone and non-convex set.

(iii) If set map $F$ is reduced to a vector map, then the quasi $S$-upper(lower) semicontinuous becomes $S$-upper(lower) semicontinuous of vector-valued mappings.

The following result can be obtained immediately.

Corollary 3.3. Let $k \in$ int $S$ and $a \in V$. Let $X_{0} \subset X$ be a nonempty compact convex subset, and let $f: X_{0} \times Y_{0} \rightarrow V$ be a vector-valued mapping. If

(i) for each $y \in X_{0}, f(x, y)$ is $S$-upper semicontinuous and $f(x, x)$ is S-lower semicontinuous;

(ii) for each $x \in X_{0}, f(x, \cdot)$ is properly $S$-quasiconvex on $X_{0}$;

(iii) $\bigcup_{x \in X_{0}} f(x, x) \subset V \backslash\{-S \backslash\{\theta\}\}$,

then the vector equilibrium problem has a solution.

Remark 3.4. The following assumption is standard in the literatures

$$
f(x, x) \in S, \quad \forall x \in X_{0} .
$$

Clearly, the corresponding assumption in Corollary 3.3 is strictly weakened. The following example illustrates this case.

Example 3.5. Let $X=R, V=R^{2}, X_{0}=[-1,1]$ and $S=R_{+}^{2}$. Let $f: X_{0} \times X_{0} \rightarrow R^{2}$ be defined by

$$
f(x, y)=(y(x, 1)) .
$$

Obviously, all conditions of Corollary 3.3 hold. By simple calculation, we have

$$
\bigcup_{x \in X_{0}} f(x, x)=\left\{(u, v): u=v^{2}, v \in[-1,1]\right\} .
$$

Clearly,

$$
\bigcup_{x \in X_{0}} f(x, x) \subset V \backslash\{-S \backslash\{\theta\}\} .
$$

Taking $\bar{x} \in[-1,0), \bar{x}$ is a solution of the vector equilibrium problem. However, let $x_{0}=-1$,

$$
f\left(x_{0}, x_{0}\right) \notin R_{+}^{2} .
$$

Hence, these results in $[1,2,3,4,5,10]$ are not applicable, however, Corollary 3.3 is still applicable.

Now, by using the Ky Fan section theorem and the separation theorem of convex sets, we prove the following result. 
Theorem 3.6. Let $X_{0} \subset X$ be nonempty compact convex subset, and let $F: X_{0} \times Y_{0} \rightarrow 2^{V}$ be a set map. If

(i) for each $y \in X_{0}, F(x, y)$ is quasi $S$-upper semicontinuous and $F(x, x)$ is quasi S-lower semicontinuous;

(ii) for each $x \in X_{0}, F(x, \cdot)$ is natural $S$-quasiconvex on $X_{0}$;

(iii) $\operatorname{co}\left(\bigcup_{x \in X_{0}} F(x, x)\right) \subset V \backslash\{-S \backslash\{\theta\}\}$,

then the SVEP has a solution.

Proof. Letting $s^{*} \in S^{*} \backslash\{\theta\}$, we prove that there exists $\bar{x} \in X_{0}$ satisfying

$$
s^{*} \circ F\left(\bar{x}, X_{0}\right) \subset \bigcup_{x \in X_{0}} s^{*} \circ F(x, x)+R_{+} .
$$

Let

$$
A:=\left\{(x, y) \in X_{0} \times X_{0}: s^{*} \circ F(x, y) \subset \bigcup_{x \in X_{0}} s^{*} \circ F(x, x)+R_{+}\right\} .
$$

By the definition of $A$, it is easy to see that, for any $y \in X_{0}$, the set $\left\{x \in X_{0}:(x, y) \in A\right\}$ is a closed set and, for any $x \in X_{0},(x, x) \in A$.

Next, we show that, for any $x \in X_{0},\left\{y \in X_{0}:(x, y) \notin A\right\}$ is convex. By the definition of $A$ and the assumptions, we have

$$
\left\{y \in X_{0}:(x, y) \notin A\right\}=\left\{y \in X_{0}: \exists z \in s^{*} \circ F(x, y) \text { s.t. } z<\min \bigcup_{x \in X_{0}} s^{*} \circ F(x, x)\right\} .
$$

Letting $y_{1}, y_{2} \in\left\{y \in X_{0}:(x, y) \notin A\right\}$ and $l \in[0,1]$, we can obtain

$$
\exists z_{1} \in s^{*} \circ F\left(x, y_{1}\right) \quad \text { s.t. } \quad z_{1}<\min \bigcup_{x \in X_{0}} s^{*} \circ F(x, x),
$$

and

$$
\exists z_{2} \in s^{*} \circ F\left(x, y_{2}\right) \quad \text { s.t. } \quad z_{2}<\min \bigcup_{x \in X_{0}} s^{*} \circ F(x, x) .
$$

By the natural $S$-quasiconvex of $F$ and the monotonically increasing property of $s^{*}$, there exists $z \in$ $s^{*} \circ F\left(l x_{1}+(1-l) x_{2}\right)$ satisfying

$$
\lambda z_{1}+(1-\lambda) z_{2} \geq z, \quad \forall \lambda \in[0,1]
$$

Hence,

$$
z<\min \bigcup_{x \in X_{0}} s^{*} \circ F(x, x),
$$

i.e.,

$$
l x_{1}+(1-l) x_{2} \in\left\{y \in X_{0}:(x, y) \notin A\right\} .
$$

Thus, by Lemma 2.8, there exists $\bar{x} \in X_{0}$ such that

$$
\{\bar{x}\} \times X_{0} \subset A,
$$

i.e., (3.3) holds.

We next prove that there exists $\bar{x} \in X_{0}$ satisfying

$$
F\left(\bar{x}, X_{0}\right) \subset \operatorname{co}\left(\bigcup_{x \in X_{0}} F(x, x)\right)+S .
$$


In fact, we suppose that

$$
z \notin \operatorname{co}\left(\bigcup_{x \in X_{0}} F(x, x)\right)+S
$$

i.e.,

$$
(z-S) \bigcap \operatorname{co}\left(\bigcup_{x \in X_{0}} F(x, x)\right)=\emptyset .
$$

Thus, by the strong separation of convex sets, there exists continuous linear function $s^{*} \neq \theta$ such that

$$
s^{*}(z-s)<s^{*}(u) \quad \forall u \in \operatorname{co}\left(\bigcup_{x \in X_{0}} F(x, x)\right), s \in S .
$$

Since (3.5) and $S$ is a cone, we have that $s^{*} \in S^{*} \backslash\{\theta\}$. Using (3.5) and setting $s=\theta$, we have

$$
s^{*}(z)<s^{*}(u) \quad \forall u \in \operatorname{co}\left(\bigcup_{x \in X_{0}} F(x, x)\right) .
$$

By (3.3), there exists $\bar{x} \in X_{0}$ satisfying

$$
s^{*}(w)>s^{*}(z), \quad \forall w \in F\left(\bar{x}, X_{0}\right) .
$$

By the monotonically increasing property of $s^{*}$, we have

$$
z \notin F\left(\bar{x}, X_{0}\right)+S \text {. }
$$

Hence,

$$
z \notin F\left(\bar{x}, X_{0}\right) .
$$

This shows that (3.4) holds. Using the proof of Theorem 3.1, we conclude that the SVEP has a solution.

Corollary 3.7. Let $X_{0} \subset X$ be a nonempty compact subset, and let $f: X_{0} \times Y_{0} \rightarrow V$ be a vector-valued map. If

(i) for each $y \in X_{0}, f(x, y)$ is $S$-upper semicontinuous and $f(x, x)$ is $S$-lower semicontinuous;

(ii) for each $x \in X_{0}, f(x, \cdot)$ is natural $S$-quasiconvex on $X_{0}$;

(iii) $\operatorname{co}\left(\bigcup_{x \in X_{0}} f(x, x)\right) \subset V \backslash\{-S \backslash\{\theta\}\}$,

then the vector equilibrium problem has a solution.

Remark 3.8. The convex hypothesis of Corollary 3.7 is different from the ones in Corollary 3.3. The following example illustrates this case.

Example 3.9. Let $X=R, V=R^{2}, X_{0}=[0,1]$ and $S=R_{+}^{2}$. Let $f: X_{0} \times X_{0} \rightarrow R^{2}$ be defined by

$$
f(x, y)=\left(x\left(y, y^{2}-2 y\right)\right) .
$$

Clearly, all the assumptions of Corollary 3.7 hold. But, $f(x, \cdot)$ is not properly $S$-quasiconvex. Thus, Corollary 3.3 are not applicable, however, Corollary 3.7 is applicable. Note that

$$
\operatorname{co}\left(\bigcup_{x \in X_{0}} f(x, x)\right) \subset V \backslash\{-S \backslash\{\theta\}\} .
$$

Taking $\bar{x} \in[0,1]$, we have that $\bar{x}$ is a solution of vector equilibrium problem. In addition, letting $x_{0} \neq 0$, we have

$$
f\left(x_{0}, x_{0}\right) \notin R_{+}^{2} .
$$

Hence, our results are different from the corresponding ones in the literatures. 
Next, we also obtain two types of existence results of the SWVEP by virtue of the approach of Theorems 3.1 and 3.6, respectively.

Theorem 3.10. Let $k \in$ int $S$, and $a \in V$. Let $X_{0} \subset X$ be a nonempty compact convex subset, and let $F: X_{0} \times Y_{0} \rightarrow 2^{V}$ be a set map. If

(i) for each $y \in X_{0}, F(x, y)$ is quasi $S$-upper semicontinuous and $F(x, x)$ is quasi S-lower semicontinuous;

(ii) for each $x \in X_{0}, F(x, \cdot)$ is properly $S$-quasiconvex on $X_{0}$;

(iii) $\bigcup_{x \in X_{0}} F(x, x) \subset V \backslash\{-$ int $S\}$,

then the SWVEP has a solution.

Proof. In view of the proof of Theorem 3.1 and $S+V \backslash\{-$ int $S\}=V \backslash\{-$ int $S\}$, one can obtain the desired conclusion immediately.

Corollary 3.11. Let $k \in$ int $S$, and $a \in V$. Let $X_{0} \subset X$ be a nonempty compact convex subset, and let $f: X_{0} \times Y_{0} \rightarrow V$ be a vector map. If

(i) for each $y \in X_{0}, f(\cdot, y)$ is $S$-upper semicontinuous and $f(x, x)$ is $S$-lower semicon-tinuous;

(ii) for each $x \in X_{0}, f(x, \cdot)$ is properly $S$-quasiconvex on $X_{0}$;

(iii) $\bigcup_{x \in X_{0}} f(x, x) \subset V \backslash\{-$ int $S\}$,

then the weak vector equilibrium problem has a solution.

All the assumptions of Example 3.5 holds. It is obvious that

$$
\bigcup_{x \in X_{0}} f(x, x) \subset V \backslash\left\{-i n t R_{+}^{2}\right\} .
$$

Taking $\bar{x} \in[-1,0)$, we have that $\bar{x}$ is a solution of the weak vector equilibrium problem. However, letting $x_{0}=-1$, we have

$$
f\left(x_{0}, x_{0}\right) \notin R_{+}^{2} .
$$

This shows that our results are different with the corresponding ones in the literatures.

Theorem 3.12. Let $X_{0} \subset X$ be nonempty compact convex subset, and let $F: X_{0} \times Y_{0} \rightarrow 2^{V}$ be a set map. If

(i) for each $y \in X_{0}, F(x, y)$ is quasi $S$-upper semicontinuous and $F(x, x)$ is quasi S-lower semicontinuous;

(ii) for each $x \in X_{0}, F(x, \cdot)$ is natural $S$-quasiconvex on $X_{0}$;

(iii) $\operatorname{co}\left(\bigcup_{x \in X_{0}} F(x, x)\right) \subset V \backslash\{-$ int $S\}$,

then the SWVEP has a solution.

Proof. Using the proof of Theorem 3.6 and $S+V \backslash\{-i n t S\}=V \backslash\{-i n t S\}$, one can obtain the desired conclusion immediately.

Corollary 3.13. Let $k \in$ int $S$, and $a \in V$. Let $X_{0} \subset X$ be a nonempty compact convex subset, and let $f: X_{0} \times Y_{0} \rightarrow V$ be a vector map. If

(i) for each $y \in X_{0}, f(\cdot, y)$ is $S$-upper semicontinuous and $f(x, x)$ is $S$-lower semicon-tinuous;

(ii) for each $x \in X_{0}, f(x, \cdot)$ is properly $S$-quasiconvex on $X_{0}$;

(iii) $\operatorname{co}\left(\bigcup_{x \in X_{0}} f(x, x)\right) \subset V \backslash\{-$ int $S\}$,

then the weak vector equilibrium problem has a solution. 
All the assumptions of Example 3.9 holds. It is obvious that

$$
\operatorname{co}\left(\bigcup_{x \in X_{0}} f(x, x)\right) \subset V \backslash\left\{-i n t R_{+}^{2}\right\} .
$$

Taking $\bar{x} \in[0,1]$, we have that $\bar{x}$ is a solution of the weak vector equilibrium problem. However, $x_{0} \neq 0$, $f\left(x_{0}, x_{0}\right) \notin R_{+}^{2}$. Therefore, our results are different with the corresponding ones in the literatures.

\section{Funding}

This research was partially supported by Yunnan Provincial Department of Education (No. 2018JS370) and National Natural Science Foundation of China (No. 11901511).

\section{Acknowledgements}

The authors are grateful to the anonymous referees for their valuable comments and suggestions, which improved the paper.

\section{REFERENCES}

[1] M. Bianchi, G. Kassay, R. Pini, Existence of equilibria via Ekeland's principle, J. Math. Anal. Appl. 305 (2005), $502-512$.

[2] G. Bigi, A. Capătă, G. Kassay, Existence results for strong vector equilibrium problems and their applications, Optimization, 61 (2012), 567-583.

[3] Q.H. Ansari, F. Flores-Bazán, Recession methods for generalized vector equilibrium problems, J. Math. Anal. Appl. 321 (2006), 132-146.

[4] Y.C. Lin, On generalized vector equilibrium problems, Nonlinear Anal. 70 (2009), 1040-1048.

[5] X.H. Gong, Efficiency and Heing efficiency for vector equilibrium problems, J. Optim. Theory Appl. 108 (2001), $139-154$.

[6] B.T. Kien, N.C. Wong, J.C. Yao, Generalized vector variational inequalities with star-pseudomonotone and discontinuous operators, Nonlinear Anal. 68 (2008), 2859-2871.

[7] S.J. Li, G. Chen, G.M. Lee, Minimax theorems for set-valued mappings, J. Optim. Theory Appl. 106 (2000), $183-200$.

[8] S.J. Li, G. Chen, X.Q. Yang, Generalized minimax inequalities for set-valued mappings, J. Math. Anal. Appl. 281 (2003), 707-723.

[9] Y. Zhang, S.J. Li, Generalized Ky Fan minimax inequalities for set-valued mappings, Fixed Point Theory, 15 (2014), 609-622.

[10] Y. Han, N.J. Huang, Existence and connectedness of solutions for generalized vector quasiequilibrium problems, J. Optim. Theory Appl. 179 (2018), 65-85.

[11] L.Q. Anh, N.V. Hung, Gap functions and Hausdorff continuity of solution mappings to parametric strong vector quasiequilibrium problems, J. Ind. Manag. Optim. 14 (2018), 65-79.

[12] L.Q. Anh, N.V. Hung, Levitin-Polyak well-posedness for strong bilevel vector equilibrium problems and applications to traffic network problems with equilibrium constraints, Positivity, 22 (2018), 1223-1239.

[13] Z.Y. Peng, Y. Zhao, X.M. Yang, Semicontinuity of approximate solution mappings to parametric set-valued weak vector equilibrium problems, Numer. Funct. Anal. Optim. 36 (2015), 481-500.

[14] J. Zeng, S.J. Li, An Ekeland's variational principle for set-valued mappings with application, J. Comput. Appl. Math. 230 (2009), 477-484.

[15] C. Gerstewitz, Nichtkonvexe trennungssätze und deren anwendung in der theorie der vektoroptimierung, Seminarberichte der Secktion Mathematik der Humboldt-Universität zu Berlin 80 (1986), 19-31.

[16] C. Certh, P. Weidner, Nonconvex separation theorems and some applications in vector optimization, J. Optim. Theory Appl. 67 (1990), 297-320.

[17] K. Fan, Minimax theorems, Proc. Natl. Acad. Sci. USA 39 (1953), $42-47$.

[18] K. Fan, Fixed-point and minimax theorems in locally convex topological linear spaces, Proc. Natl. Acad. Sci. USA. 38 (1952), 121-126. 